\section{BLOOD TRANSFUSION AT THE FRONT AREA *}

BENJAMIN I. HARRISON, M.D. (Cleveland) Lieutenant, M. R. C., U. S. Army

FRANCE

This paper is based on observations of transfusions of blood performed at advanced hospitals. The patients were those having but little prospect of recovery otherwise.
Donors.-Arrangements were made through the medical officer, with the sergeant in charge of the walking wounded, to supply lightly wounded men for donors. As the occasion required, those selected were sent to the resuscitation ward for grouping and charting. A grouped list of all donors was available at a minute's notice, day or night. A list of about ten donors, usually assigned to light duty around nearby wards, was kept at all times. As economy we used Group II, III and IV men. Some casualty clearing stations use only Group IV donors, and do not group

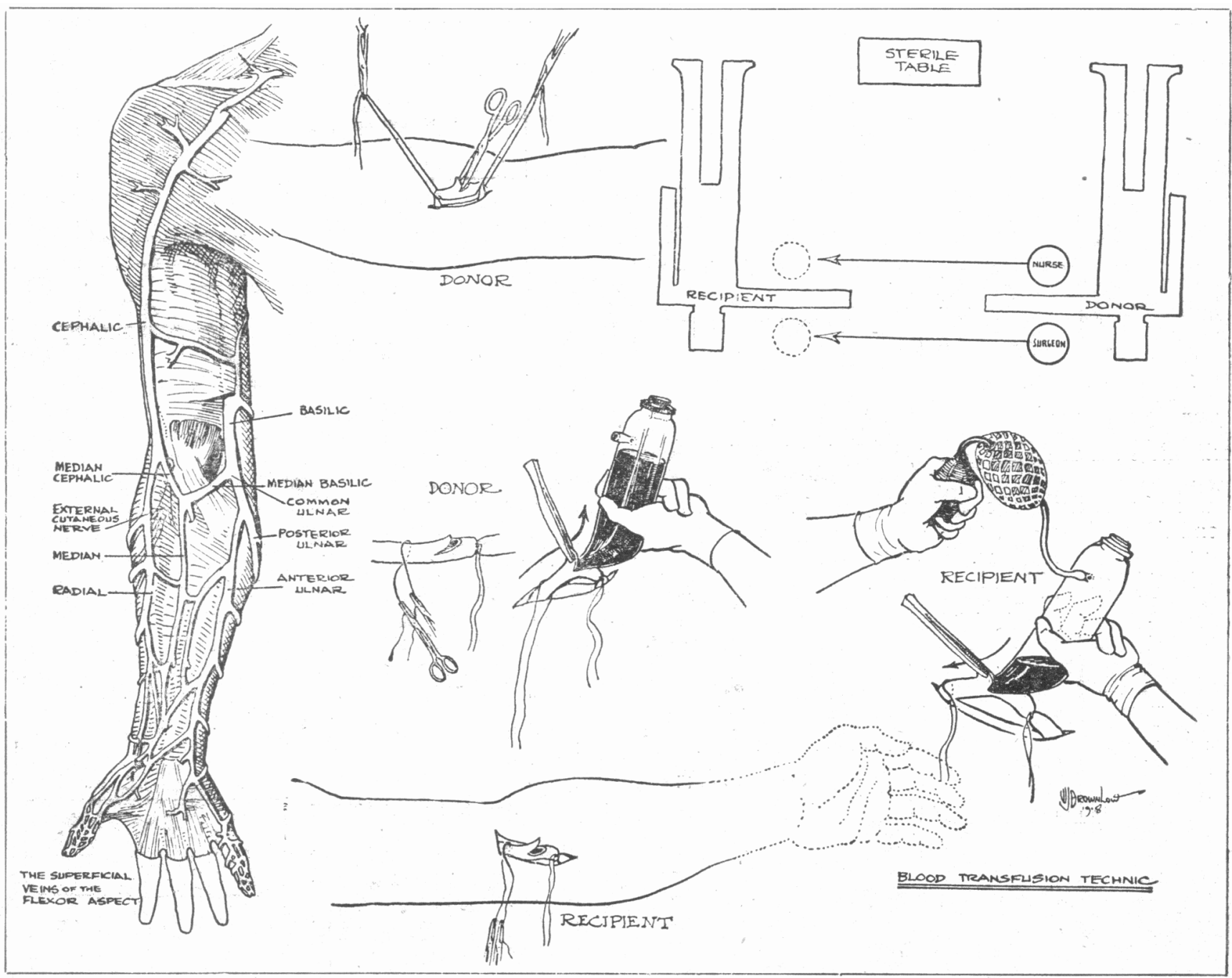

Fig. 1.-Technic of blood transfusion.

Grouping.-The selection of donors and the grouping of recipients was done by the method described by Lee, ${ }^{1}$ with serums and necessary equipment furnished by Captain Karsner. ${ }^{2}$ By holding the slide over a candle, the reactions were judged with the naked eye. All cases were classified without difficulty. The technic was so simple that it could be carried out in the resuscitation ward, proving very practical from all points of view.

* From No. 9 (Lakeside, U. S. Army) General Hospital.

1. Lee, R. I. A Simple and Rapid Method for the Selection of Suitable Donors for Transfusion by the Determination of Blood Ciroups, British Medical Journal, 1917, 2, 684

2. Karsner, H. T.: Transfusion with Tested Bloods, Including the Frouping of One Thousand Bloods and a Method for Use at Advanced liospitals, Tue Journal. A. M. A., March 16, 1918, p. 769. their patients; however, we found that it required about ten minutes to group a patient, and thus, we were able to use all donors excepting the group I men.

Technic of Transfusion.--The indirect method of transfusion was used, with the technic as shown in Figure 1. The tubes were coated with ordinary hard paraffin obtained from the pharmacy at the casualty clearing station.

A vein of the flexor aspect of the arm was used, 3 c.c. of 1 per cent. procain being used for infiltration anesthesia. The proximal end of the donor's vein was tied, and the distal end of the recipient's, a ligature loop being placed around the distal end of the donor's vein and the proximal end of the recipient's. Traction by means of a hemostat closed the veins. 
In our series of cases, there was not an instance of coagulation in the tube. The average time for filling each tube of 300 c.c. capacity was three minutes; for the entire transfusion, approximately thirty minutes.

Differential Diagnosis-Hemorrhage and Shock.From a clinical point of view, it is very difficult, in some cases, to know whether a patient is suffering from

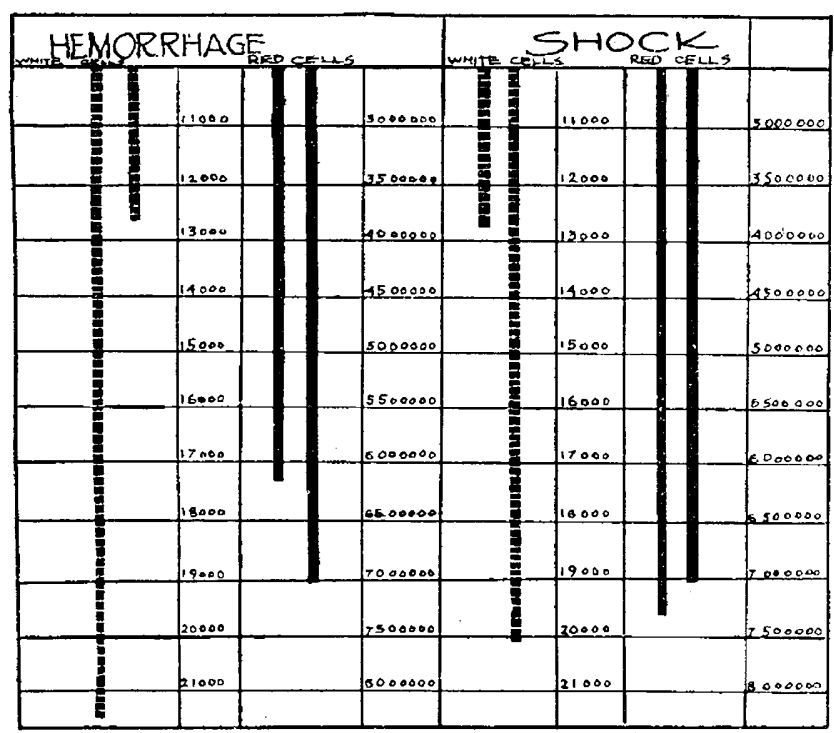

Fig. 2.- Blood counts in shock and hemorrhage. (Crile, G. W.: Hemorrbage and Transfusion, p. 111.)

hemorrhage, from shock, or from both. In the three conditions, the pulse, the blood pressure and the collapse are not dissimilar. In hemorrhage, however, the patient is generally restless and anxious or worried about his condition. A shock patient, on the other hand, is inclined to be listless and apathetic. In extreme cases of shock, the patient is almost invariably

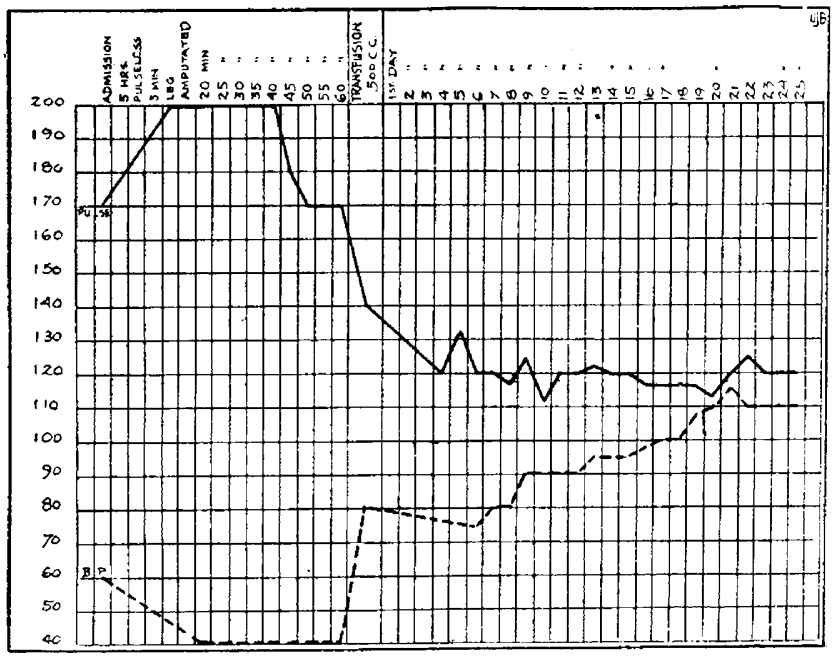

Fig. 3.-Curve of pulse and blood pressure in transfusion for !lem. orrhage.

of a grayish-blue color, this symptom being attributed by Crile to a failure of internal respiration.

As a practical measure, I would suggest that a note be made on the field card by the medical officer at the field ambulance, indicating the amount of hemorrhage.

Increase of White Blood Cells in Hemorrhage.-In hemorrhage, we found a definite increase in the white blood count, confirming the observation of Crile. $^{3}$ However, our series showed a blood count, in severe cases, amunting to 45,000 . In shock, on the other hand, we found no increase in the white blood count. Nearly all our cases were examined under eight hours after injury (Fig. 2).

Transfusion for Hemorrhage. - In acute hemorrhage, transfusion of blood is specific, provided the patient has not been in an exsanguinated state long enough to cause irreparable injury to the brain. The effects generally are striking, immediate and permanent. In our series, we had only one case in which transfusion was useless, owing to this fact.

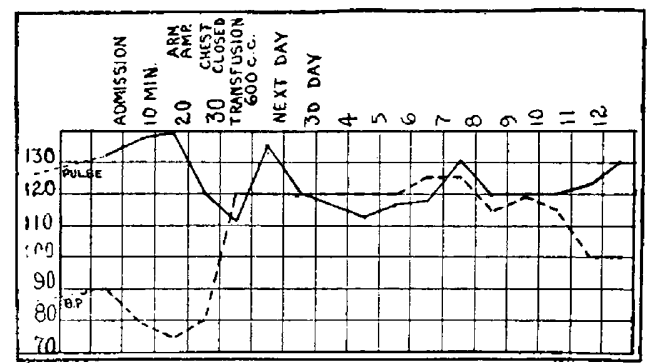

Fig. 4.-Curve of pulse and blood pressure in transfusion for shuck and hemorrhage.

Transfusion of blood is followed by a marked rise of blood pressure, increase in pulse volume with slowing of rate, while respiration returns to normal (Fig. 3).

Transfusion for Hemorrhage and Shock.-In cases in which there was both hemorrhage and shock, transfusion proved effective, but the results were not as good as those following transfusion for hemorrhage alone (Fig. 4).

Transfusion for Shock.-In shock without hemorrhage, if the patient had not yet reached the gray-blue stage, a certain amount of benefit followed transfusion of blood-a change often sufficient to carry through operation to recovery. If, however, the patient had reached the gray-blue stage, no benefit resulted from transfusion (Fig. 5).

In a series of fifty transfusions, in cases in which recovery by the usual course was not expected, 55 per cent. of the patients recovered. Two of the patients died of pneumonia. In several, with gunshot wounds of the abdomen, transfusion was of no avail. In a few of the most severe cases of gunshot wounds of the abdomen we gave 200 c.c. of

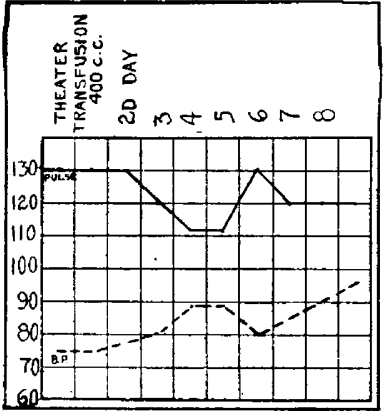

Fig. 5. - Curve of pulse and blood pressure in transfusion for shock. blood three days in succession, following the primary transfusion. However, this treatment showed no more improvement than in those cases in which transfusion was performed at one séance, provided sufficient blood had been given at the primary transfusion.

Only once was there a slight reaction. This maii underwent transfusion for hemorrhage from a severed

3. Crile, G. W.: Hemorrhage and Transfusion, New York, D. Appleton \& Co., 1909 , p. 111 . 
popliteal artery. Fourteen days later the leg was amputated. A severe hemorrhage followed the operation, so that the man was again transfused. On the third day, a slight rash, not unlike serum rash, had developed, accompanied by a temperature of $101 \mathrm{~F}$. The following day the temperature was down to normal, and the rash cleared up without further disturbance.

\section{CONCLUSIONS}

1. Timely blood transfusion for hemorrhage is a specific cure.

2. For hemorrhage and shock, results are good, but not specific.

3. For pure shock early transfusion, that is, transfusion before the "gray-blue" stage is reached, is use$\mathrm{ful}$, and is the most efficient treatment. Transfusion in the gray-blue stage is of little use. In this class, no other treatment has been useful.

\section{FURTHER EXPERIENCE WITH FACE MASKS *}

\section{GEORGE H. WEAVER, M.D. CHICAGO}

When the Durand Hospital of the John McCormick Institute for Infectious Diseases was opened, rigid aseptic technic was adopted and the nurses were specially instructed in measures calculated to protect them from infections. From March 12, 1913, to Nov. 1, 1914, nine out of sixty-nine nurses, or 13 per cent., acquired clinical diphtheria. From this time on, all nurses giving a positive Schick test were immunized with diphtheria antitoxin. This practically eliminated active diphtheria; but from Nov. 1, 1914, to June 1, 1916, weekly throat cultures disclosed ten diphtheria bacillus carriers among forty-three nurses, or 23.25 per cent.

Up to June 1, 1916, nine cases of scarlet fever occurred among 112 nurses who were on duty, or in 8 per cent.

As we were unable to explain so many instances of infection through faulty technic, an effort was made to eliminate a possible factor of danger that had previously been largely ignored, namely, infection through mouth spray. Since June 1, 1916, gauze masks have been used by the nurses, and up to Oct. 1, 1918, six diphtheria bacillus carriers have been detected among seventy-three nurses, or 5.2 per cent. ${ }^{1}$ No case of scarlet fever has occurred since masks were worn. The nurses are instructed to change the mask as soon as it has been known to be grossly contaminated, and never to put the hands to the mask to adjust it, etc., until the hands have been thoroughly washed.

Early in 1918, bacteriologic tests showed that the masks we were using did not remove all the bacteria thrown out in mouth spray. The masks consisted of two layers of gauze, 28 by 24 mesh; but as they were worn only once before washing and resterilizing, shrinkage soon made the openings in the gauze much closer than they were in the new masks.

* From the John McCormick Institute for Infectious Diseases.

1. Our experiences up to Dec. 1, 1917, were reported by Weaver, G. H.: The Value of the Face Mask and Other Measures in Prevention of Diphtheria, Meningitis, Pneumonia, etc., THE Journal A. M. A., Jan. 12,1918 , p. 76 .
Studies were instituted to learn how the masks could be made most efficient. The results that we have obtained agree very well with those recently reported by Haller and Colwell ${ }^{2}$ and Doust and Lyon. ${ }^{3}$ They are here presented as serving further to emphasize the importance of gauze masks of proper composition. When the probable importance of droplet infection in the dissemination of human tuberculosis attracted attention, gauze masks to be worn by the patient were advocated. Hamilton, in 1905, advised the use of gauze masks to cover the mouth of patients with scarlet fever when there were severe streptococcal complications and when the individual could not be properly isolated. In 1916, Meltzer advocated the use of a fine mesh net over the faces of patients with infantile paralysis and also over the faces of attendants. Various mechanical protections of the face were formerly used by physicians when swabbing throats and doing tracheotomy in cases of diphtheria. Gauze masks have been long used by many surgeons and their assistants with the purpose of protecting wounds from infection by mouth droplets.

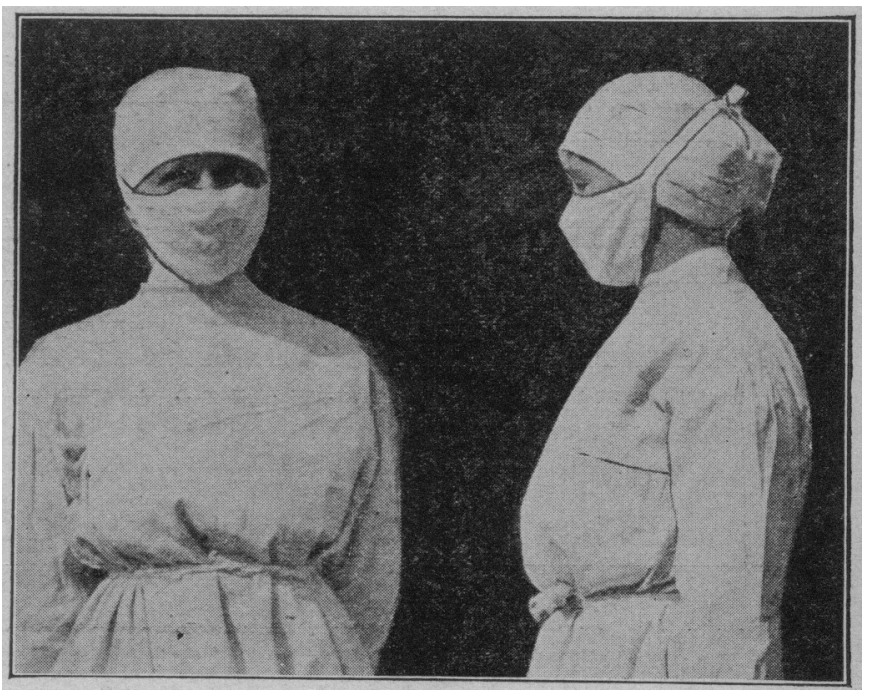

Durand hospital mask, devised by Miss Charlotte Johnson, superin. tendent: The gauze ( 44 by 40 mesh) is cut 8 inches wide and 23 inches long. The sides and one end are turned down one-quarter inch. It is folded twice, the unturned end first, making a $71 / 2$-inch square. The opposite diagonal corners are cut off 1 inch and the raw edge is turned in one-half inch. It is stitched firmly all around. A 1 -inch dart $11 / 2$ inches long is taken up at the middle of each side of the mask. A 14-inch tape is sewed on the opposite uncut corners. This mask has the advantage of covering the nose and mouth and in making the traction on the chin and not drawing on the nose and lips.

Our experiments were performed in a quiet room with no currents of air. It was assumed that the power of various gauzes to filter moist spray from air would increase with closeness of mesh and with the number of layers employed. In the first tests a spray of carbolfuchsin was employed, the dye being susceptible of fairly accurate measurements. It was thrown as spray by a hand atomizer. Briefly, these tests showed that the percentage of fuchsin passing through the gauze becomes progressively less as the mesh of the gauze becomes closer and as the number of layers of gauze is multiplied.

2. Haller, D. A., and Colwell, R. C.: The Protective Qualities of the Gauze Face Mask, The Journal A. M. A., Oct. 12, 1918, p. 1213. 3. Doust, B. C., and Lyon, A. B.: Face Masks in Infections of the Respiratory Tract, The Journal A. M. A., Oct. 12, 1918, p. 1216. 